\title{
Estado da arte - estudo sobre mulheres gestoras na educação: o que dizem as produções
}

\author{
State of art - study about Women Managers in Education: what researches say
}

Keoma Yoshio de Paula Bordinhão Mestranda em Educação Pontifícia Universidade Católica do Paraná - PUCPR Curitiba, Paraná - Brasil. k.keoma@hotmail.com

Marlene Oliveira Doutoranda em Educação Instituto Federal do Paraná - IFPR Curitiba, Paraná - Brasil. Marlene.oliveira@ifpr.edu.br

Rosa Lydia Teixeira Corrêa Doutora em História Econômica pela Universidade de São Paulo (USP) Pontifícia Universidade Católica do Paraná - PUCPR Curitiba, Paraná - Brasil. rosa_lydia@uol.com.br

\begin{abstract}
Resumo: A mulher foi conquistando, vagarosamente, vários espaços sociais por meio do ingresso na escola, visando ampliar a educação voltada aos cuidados do lar e da família. Progressivamente, foram garantidos o direito de instrução em diferentes níveis de escolarização, inclusive a profissionalização do magistério, chegando a cargos de gestoras educacionais. Incialmente, em conventos vinculados a congregações religiosas católicas e como líderes de outras profissões religiosas, mundo à fora. Gradativamente, as mulheres se propuseram a exercer funções e cargos há muito de exclusividade masculina. Este artigo tem por objetivo identificar estudos sobre mulheres gestoras, contidos em teses e dissertações nos bancos de dados da CAPES e BDTD, no período de 2014 a 2019. Metodologicamente, trata-se de uma pesquisa do tipo estado da arte, com fundamentação em Romanowski e Ens (2006). Os resultados encontrados se dividem entre o campo de conhecimento da administração e da educação em nível superior, reportando-se ao Ensino Superior.
\end{abstract}

Palavras chave: educação; história da educação; mulheres gestoras.

\begin{abstract}
Slowly, women have conquered various social spaces through entering schools, looking forward to extending their education surrounding caring for their homes and families. Progressively, the right to instruction on different schooling levels was granted, including the professionalization of the teacher-training college. That originated the function of educational managers, initially on convents linked to Catholic religious congregations, and as leaders of other religious professions, around the world. Gradually, women decided to undertake functions that were traditionally exclusive to men. This article aims to identify studies regarding women managers, included on theses and dissertations from CAPES' and BDTD's database, within the time frame of 2014 to 2019. Methodologically, this is a state of art type research, based on Romanowski and Ens (2006). The results found are divided between the knowledge areas of administration and of higher education, reporting themselves to Higher Education.
\end{abstract}

Keywords: education; history of education; women managers.

Cite como

\section{(ABNT NBR 6023:2018)}

BORDINHÃO, Keoma Yoshio de Paula; OLIVEIRA, Marlene; CORRÊA, Rosa Lydia Teixeira. Estado da arte - estudo sobre mulheres gestoras na educação: o que dizem as produções. Dialogia, São Paulo, n. 40, p. 1-13, e20488, jan./abr. 2022. Disponível em: https://doi.org/10.5585/40.2021.20488.

American Psychological Association (APA)

Bordinhão, K. Y. de. P., Oliveira, M., \& Corrêa, R. L. T. (2022, jan./abr.) Estado da arte - estudo sobre mulheres gestoras na educação: o que dizem as produções. Dialogia, São Paulo, 40, p. 1-13, e20488. https://doi.org/10.5585/40.2021.20488. 


\section{Introdução}

De subalterna ao poder público, de coadjuvante a protagonista, pensar como se deu o percurso da mulher na história, suas lutas e conquistas torna-se importante para a sociedade contemporânea a partir do momento em que se põe em evidência reflexões sobre os caminhos trilhados até o momento, sem perder de vista a subjetividade feminina, o eu mulher.

A mulher, ao longo de um processo histórico e por muito tempo, se manteve invisível aos olhos da sociedade, como afirma Perrot (2017, p. 170) ao dizer que "da História, muitas vezes a mulher é excluída". Involuntariamente, o papel da mulher demarcou por anos a submissão ao patriarcado. A construção de uma identidade se deu por meio de grandes entraves sociais e culturais, como também políticos, repercutidos por meio de movimentos diferentes em busca de ascensão social, desde o Brasil Colônia até a atualidade.

A ascensão feminina foi conquistando, vagarosamente, vários espaços sociais, destacando seu ingresso na escola, considerado tardio e com início de formação voltada aos cuidados do lar e da família. Progressivamente e com o apoio de aportes legais, foi garantido o direito de instrução em diferentes níveis de escolarização, inclusive a profissionalização do magistério, chegando aos cargos de gestoras educacionais. Incialmente, em conventos vinculados a congregações religiosas católicas e como líderes de outras profissões religiosas, mundo à fora.

Gradativamente, as mulheres se propuseram a exercer funções e cargos que até o momento eram exclusivamente masculinas. Isso se configurava também nas instituições de ensino, onde, mesmo sendo maioria, as mulheres se sujeitam às ordens advindas de seus superiores, em sua maioria, masculinos.

Com a formação de uma nova identidade feminina em múltiplas instâncias sociais, a luta pela obtenção de direitos e pelo reconhecimento como ser social foi ganhando espaço no decorrer dos anos. Hoje, um número considerável de mulheres desenvolve funções tanto na docência, como na gestão de instituições de ensino municipais, estaduais e federais. Mesmo sabendo que muito ainda se tem a conquistar, enaltecemos seu percurso histórico de equidade e igualdade de acesso e inserção social mais amplos.

Tais avanços norteiam o estudo feito por meio de levantamento do tipo "estado da arte", entendido como "uma exposição sobre o nível de conhecimento e desenvolvimento de um campo ou questão" (SPINK, 1996, p. 167) acerca das discussões presentes nas produções acadêmicas a respeito da temática mulher gestora educacional, servindo como uma espécie de termômetro para a análise dessa temática e que se acha em processo de desenvolvimento. Nesse sentido, o presente ensaio tem por objetivo identificar como pesquisas estão abordando esse assunto nas teses e nas 
dissertações localizadas nos bancos de dados da Coordenação de Aperfeiçoamento de Nível Superior (CAPES) e da Biblioteca Digital Brasileira de Teses e Dissertações (BDTD), no período de 2014 a 2019, portanto, nos últimos cinco anos, o que se justifica em decorrência de o estudo que origina esse levantamento estar situado nesse período.

Dada a importância de valorizar essa temática de conquistas, é imprescindível analisar e refletir a respeito desse assunto pelo fato de tudo indicar serem poucos os cargos de gestão exercidos por mulheres. Assim, o interesse do presente estudo volta-se especificamente para mulheres gestoras que atuaram em instituições educacionais. Desse modo indagamos: $\mathrm{O}$ que indicam os estudos acerca desse tema?

Essa mesma mulher que rompeu com paradigmas sociais historicamente postos cursou o Ensino Superior e protagonizou-se gestora de uma instituição de ensino, transpondo com o subalterno.

\section{Estado da arte como metodologia de pesquisa}

A metodologia utilizada para este estudo foi a pesquisa qualitativa, do tipo exploratória, com base em documentos de domínio público e da literatura científica, proporcionando ao pesquisador se familiarizar com o objeto de estudo.

Assim, para avançarmos no estudo em relação às mulheres gestoras educacionais, organizamos no primeiro semestre de 2020 uma busca minuciosa por meio da pesquisa tipo “estado da arte", nas produções científicas brasileiras limitadas ao recorte temporal de 2014 a 2019, como indicado anteriormente, e que se reportassem em algum momento aos descritores preestabelecidos: mulheres gestoras; mulheres gestoras na educação; mulheres gestoras na história da educação; mulheres gestoras na instituição de ensino.

Os descritores foram sendo delimitados e ampliados gradativamente, considerando a especificidade do objeto de estudo, já que este está situado no campo da história da educação, porém sem caracterizar o espaço de atuação, e passando pelo contexto da educação, ainda na intenção de encontrar alguma publicação na história da educação, e, por fim, restringir a instituições escolares.

Desse modo, utilizou-se como referência para o levantamento dos dados as bases científicas da Coordenação de Aperfeiçoamento de Nível Superior (CAPES) e da Biblioteca Digital Brasileira de Teses e Dissertações (BDTD). De caráter bibliográfico, a pesquisa tipo "estado da arte”, ou também conhecida como "estado do conhecimento", tem por objetivo mapear e/ou investigar inúmeros campos do conhecimento que serão objetos de análise de acordo com suas produções 
(FERREIRA, 2002). Ela possibilita ao pesquisador possuir mecanismos preliminares de determinado objeto de estudo, podendo examinar diferentes aspectos, como descrevem Romanowski e Ens (2006, p. 39), que também a classificam como “estudos de revisão:

Essas análises possibilitam examinar as ênfases e temas abordados nas pesquisas; os referenciais teóricos que subsidiaram as investigações; a relação entre o pesquisador e a prática pedagógica; as sugestões e proposições apresentadas pelos pesquisadores; as contribuições da pesquisa para mudança e inovações da prática pedagógica; a contribuição dos professores/pesquisadores na definição das tendências do campo de formação de professores.

O mapeamento dos periódicos, além de manifestar a carência de pesquisas por determinados objetos, contribui para dar continuidade à investigação, ciente que permite obter análise do que tem se referenciado nas produções acadêmicas, assim como apontar possíveis caminhos para prosseguir com futuras pesquisas, fornecendo novos subsídios de estudos e oferecendo um leque de possibilidades. Sendo assim, para Romanowski e Ens (2006, p. 41), a pesquisa tipo “estado da arte” prevê:

uma visão geral do que vem sendo produzido na área e uma ordenação que permite aos interessados perceberem a evolução das pesquisas na área, bem como suas características e foco, além de identificar as lacunas ainda existentes.

Para realizar este estudo, adotou-se os seguintes procedimentos, recomendados por Romanowski (2002, p. 15-16):

\begin{abstract}
a) definição dos descritores para direcionar as buscas a serem realizadas; b) localização dos bancos de pesquisas, teses e dissertações, catálogos e acervos de bibliotecas, biblioteca eletrônica que possam proporcionar acesso a coleções de periódicos, assim como aos textos completos dos artigos; c) estabelecimento de critérios para a seleção do material que compõe o corpus do estado da arte; d) levantamento de teses e dissertações catalogadas. e) leitura das publicações com elaboração de síntese preliminar, considerando o tema, os objetivos, as problemáticas, metodologias, conclusões, e a relação entre o pesquisador e a área. (grifo do original)
\end{abstract}

Nessa perspectiva, respeitando os pressupostos que a metodologia emprega, o estudo preliminar foi desenvolvido a fim de explorar produções acadêmicas que abordam a temática em relação às mulheres gestoras, possibilitando reflexões e discussões. Para sistematizar os periódicos encontrados, personificou-se as produções encontradas por base de dados: BDTD e CAPES. Para tanto, dois filtros foram utilizados em ambas: período (2014 a 2019) e o tipo de documento (teses e dissertações). 


\section{O que indicam as publicações}

Os resultados da investigação foram demonstrados em quadros seguindo a ordem das indicações: Tipo (Tese ou Dissertação), Autoria, Ano de publicação, Título e as Palavras-chave do resumo. Iniciando pela base de dados BDTD, ao buscar pelo descritor "Mulheres Gestoras", foram localizadas cinco publicações, sendo três teses e duas dissertações, como demonstrado no Quadro 1.

Quadro 1 - Pesquisas científicas encontradas com o descritor "mulheres gestoras" - BDTD (2014-2019)

\begin{tabular}{|c|c|c|c|c|c|}
\hline N. ${ }^{\circ}$ & TIPO & AUTORIA & ANO & TÍTULO & PALAVRAS-CHAVE \\
\hline 1 & Tese & $\begin{array}{l}\text { MORAES, Ana } \\
\text { Flávia de Moraes. }\end{array}$ & 2014 & $\begin{array}{l}\text { Estresse Ocupacional: um } \\
\text { olhar sobre o trabalho da } \\
\text { mulher gestora do polo } \\
\text { industrial de Manaus. }\end{array}$ & $\begin{array}{l}\text { Estresse ocupacional; } \\
\text { Tensão no trabalho; } \\
\text { Mulheres gestoras; Polo } \\
\text { Industrial de Manaus. }\end{array}$ \\
\hline 2 & Dissertação & $\begin{array}{l}\text { SILVA, Kátia } \\
\text { Elaine de } \\
\text { Vasconcelos e. }\end{array}$ & 2018 & $\begin{array}{l}\text { A representatividade das } \\
\text { mulheres na gestão } \\
\text { acadêmica da Universidade } \\
\text { Federal de Pernambuco. }\end{array}$ & $\begin{array}{l}\text { Mulheres gestoras; } \\
\text { Relações de gênero; } \\
\text { Universidade Federal de } \\
\text { Pernambuco; Metáfora } \\
\text { teto de vidro e firewall. }\end{array}$ \\
\hline 3 & Dissertação & $\begin{array}{l}\text { SILVA, Eliane } \\
\text { Vanildo da. }\end{array}$ & 2019 & $\begin{array}{l}\text { Gênero e Ensino Superior: } \\
\text { a inserção das mulheres nos } \\
\text { cursos de Engenharias da } \\
\text { UNESC. }\end{array}$ & $\begin{array}{l}\text { Mulheres; Ensino } \\
\text { Superior de Engenharia; } \\
\text { Relações de Gênero; } \\
\text { Desigualdades de } \\
\text { Gênero. }\end{array}$ \\
\hline 4 & Tese & $\begin{array}{l}\text { PIMENTA, } \\
\text { Pauline Freire. }\end{array}$ & 2019 & $\begin{array}{l}\text { Lugar de mulher é na } \\
\text { reitoria: análise discursivo- } \\
\text { crítica das formações } \\
\text { identitárias e das relações de } \\
\text { poder de mulheres do alto } \\
\text { escalão nas IFES mineiras. }\end{array}$ & $\begin{array}{l}\text { Análise de Discurso } \\
\text { Crítica; Mulher } \\
\text { Gestora; Identidade; } \\
\text { Gênero; Poder. }\end{array}$ \\
\hline 5 & Tese & $\begin{array}{l}\text { HERRERA, Vânia } \\
\text { Érica. }\end{array}$ & 2019 & $\begin{array}{l}\text { A vitrine da inclusão e o } \\
\text { espetáculo de Nicolau: a } \\
\text { ascensão profissional da } \\
\text { mulher acadêmica em } \\
\text { cargos de gestão em } \\
\text { instituições de ensino } \\
\text { superior no Brasil. }\end{array}$ & $\begin{array}{l}\text { Mulheres; Barreiras de } \\
\text { gênero; Vitrine da } \\
\text { Inclusão; Espetáculo de } \\
\text { Nicolau; Gestão do } \\
\text { Ensino Superior. }\end{array}$ \\
\hline
\end{tabular}

Fonte: Site BDTD, organizado pelas autoras, 2021.

Ao analisar os resumos das publicações, pode-se observar que são pertencentes a diferentes campos de conhecimentos, enfatizando áreas empresariais. Em geral, as pesquisas mencionam a desigualdade e a representatividade de gênero, por meio da atuação da mulher na gestão de empresas e do Ensino Superior, além do estresse como consequência do cargo exercido. Este último, salientado na tese de Moraes (2014), relata o estresse desenvolvido em ambientes industriais em decorrência da realização de várias atividades ao mesmo tempo, em diferentes graus de 
complexidade e alto grau de cobrança. Ressalta, ainda, as possibilidades e os mecanismos de enfrentamento desses problemas, como a redução da jornada de trabalho.

Uma questão muito importante e que acompanha o sexo feminino há centenas de anos é a desigualdade de gênero e o preconceito. Por mais garantias de direitos conquistados, ainda é cristalino para a sociedade a disparidade entre os sexos. Silva (2019), em sua dissertação, buscou entender o acesso e a permanência de mulheres no Ensino Superior em um curso tradicionalmente masculino. Sua investigação identificou que muito se tem a fazer para garantir a equidade entre homens e mulheres, cita ainda a importância de legislações para o fortalecimento da igualdade e que mesmo assim a discriminação, a desigualdade e o preconceito ainda se manifestavam durante as relações.

Identificamos, também, três estudos relacionados à ascensão da mulher em cargos de gestão em instituições federais do Ensino Superior. Silva (2018) analisa o acesso das servidoras na Universidade Federal de Pernambuco (UFPE) aos cargos de gestão. Alega-se um favorecimento quantitativo das mulheres docentes em comparação ao sexo oposto, mesmo assim os homens assumem mais cargos de gestão, sendo nítido a desigualdade de gênero. Em sua tese de doutorado, Herrera (2019) analisa essas barreiras que surgem na ascensão feminina, as quais dificultam o progresso profissional das mulheres.

Por mais que muitas vezes as mulheres se façam presentes em sua maioria nas instituições de Ensino Superior em nível federal, a considerar os resultados encontrados, a ocupação em cargos de gestão é estreitada por inúmeros motivos, permitindo pensar a relação de poder e de gênero. A criação de identidade feminina nesse campo de atuação não é o suficiente para romper com paradigmas preconceituosos. Por mais capacitadas que sejam as mulheres, ainda assim são ignoradas como seres capazes de gerir uma instituição. Assim como retrata Pimenta (2019) no título de sua tese Lugar de mulher é na reitoria.

Mesmo não sendo possível identificar produções em nível municipal, foi necessário trazer os estudos encontrados, vendo que as questões relatadas pelas autoras perpassam todos os níveis de ensino ou de atuação profissional feminina.

Ao analisar os resumos das publicações, pode-se observar que são pertencentes a diferentes campos do conhecimento, não se enquadrando diretamente no campo da educação, apesar de alguns estudos se referirem a gestoras do Ensino Superior. Em geral, as pesquisas mencionam a desigualdade e a representatividade de gênero no Ensino Superior por meio da atuação da mulher na gestão; apresentam questões relacionadas à ascensão da mulher em cargos de gestão em 
instituições do Ensino Superior; salientam o estresse desenvolvido em gestão de ambientes industriais e as possibilidades de enfrentamento dessas situações.

Ao buscar pelos outros três descritores, Mulheres gestoras na educação; Mulheres gestoras na história da educação; Mulheres gestoras na instituição de ensino, não foi possível localizar nenhum trabalho. Mesmo assim, a plataforma da base sugeriu tentar com outras variações na ortografia, o que não aderimos.

Iniciou-se, então, a busca na próxima base de dados, a CAPES, utilizando a mesma sequência de descritores. Com o descritor Mulheres gestoras, a princípio identificamos 28 resultados. Aplicando os filtros de tipo (teses e dissertações) e ano de defesa (2014 a 2019), obtivemos 20 resultados, contemplando novamente inúmeros campos do conhecimento.

Explorando os resumos dos resultados encontrados, selecionamos nove títulos que enfatizaram o descritor "mulheres gestoras" na pesquisa pela relevância. Sendo eles apresentados no Quadro 2.

Quadro 2 - Pesquisas científicas encontradas com o descritor "mulheres gestoras" - CAPES (2014-2019)

\begin{tabular}{|c|c|c|c|c|c|}
\hline $\mathbf{N}^{\circ}{ }^{\circ}$ & TIPO & AUTOR & ANO & TÍTULO & $\begin{array}{l}\text { PALAVRAS- } \\
\text { CHAVE }\end{array}$ \\
\hline 1 & Tese & $\begin{array}{l}\text { MORAES, Ana Flávia } \\
\text { de Moraes }\end{array}$ & 2014 & $\begin{array}{l}\text { Estresse ocupacional: um } \\
\text { olhar sobre o trabalho da } \\
\text { mulher gestora do polo } \\
\text { industrial de Manaus. }\end{array}$ & $\begin{array}{l}\text { Estresse ocupacional; } \\
\text { Tensão no trabalho; } \\
\text { Mulheres gestoras; } \\
\text { Polo Industrial de } \\
\text { Manaus. }\end{array}$ \\
\hline 2 & Dissertação & $\begin{array}{l}\text { MAFRA, Adriana } \\
\text { Pereira Santos }\end{array}$ & 2015 & $\begin{array}{l}\text { Estresse no trabalho: estudo } \\
\text { com gestoras do comércio } \\
\text { varejista na região } \\
\text { metropolitana de Belo } \\
\text { Horizonte. }\end{array}$ & $\begin{array}{l}\text { Estresse ocupacional; } \\
\text { Tensão no trabalho; } \\
\text { Mulheres gestoras; } \\
\text { Comércio varejista. }\end{array}$ \\
\hline 3 & Dissertação & $\begin{array}{l}\text { VIANA, Elisete } \\
\text { Pereira Gonçalves }\end{array}$ & 2015 & $\begin{array}{l}\text { Estresse ocupacional: estudo } \\
\text { com mulheres gerentes que } \\
\text { atuam em instituições } \\
\text { bancárias na cidade de Belo } \\
\text { horizonte/MG. }\end{array}$ & $\begin{array}{l}\text { Ocupacional; } \\
\text { Instituição bancária; } \\
\text { Mulheres gerentes. }\end{array}$ \\
\hline 4 & Dissertação & AZEVEDO, Erika & 2015 & $\begin{array}{l}\text { Estresse no trabalho: estudo } \\
\text { com gestoras de } \\
\text { organizações privadas do sul } \\
\text { do estado de Minas Gerais. }\end{array}$ & $\begin{array}{l}\text { Estresse no trabalho; } \\
\text { Estresse ocupacional; } \\
\text { Estresse em gestoras; } \\
\text { Função gerencial; } \\
\text { Mulheres gestoras. }\end{array}$ \\
\hline
\end{tabular}




\begin{tabular}{|c|c|c|c|c|c|}
\hline N. ${ }^{\circ}$ & TIPO & AUTOR & ANC & TÍTULO & PALAVRAS- \\
\hline 5 & Dissertação & $\begin{array}{l}\text { CARNEIRO, } \\
\text { Luziberto Barrozo }\end{array}$ & 2018 & $\begin{array}{l}\text { Teto de vidro: um estudo } \\
\text { sobre os fatores deste } \\
\text { fenômeno no Brasil sob a } \\
\text { percepção das mulheres } \\
\text { gestoras. }\end{array}$ & $\begin{array}{l}\text { Glass ceiling; Gestão; } \\
\text { Gênero; Cultura; } \\
\text { organizacional. }\end{array}$ \\
\hline 6 & Dissertação & $\begin{array}{l}\text { TEIXEIRA, Marcella } \\
\text { Barbosa Miranda }\end{array}$ & 2018 & $\begin{array}{l}\text { Professoras-gestoras na } \\
\text { universidade: Reflexões a } \\
\text { partir das Trajetórias de Vida } \\
\text { de Maria Carmem e Gaia. }\end{array}$ & $\begin{array}{l}\text { Mulheres e Trabalho; } \\
\text { Feminismo; Gênero; } \\
\text { Gestão Universitária. }\end{array}$ \\
\hline 7 & $\begin{array}{l}\text { Tese } \\
\text { *o trabalho }_{\text {não possui }} \\
\text { divulgação } \\
\text { autorizada }\end{array}$ & $\begin{array}{l}\text { BIASOLI, Patricia } \\
\text { Klaser }\end{array}$ & 2018 & $\begin{array}{l}\text { Mulheres em cargos de } \\
\text { gestão: conciliações, } \\
\text { dificuldades e conquistas. }\end{array}$ & \\
\hline 8 & Tese & $\begin{array}{l}\text { PIMENTA, Pauline } \\
\text { Freire }\end{array}$ & 2019 & $\begin{array}{l}\text { Lugar de mulher é na } \\
\text { reitoria: análise discursivo- } \\
\text { crítica das formações } \\
\text { identitárias e das relações de } \\
\text { poder de mulheres do alto } \\
\text { escalão nas IFES mineiras. }\end{array}$ & $\begin{array}{l}\text { Análise de Discurso } \\
\text { Crítica; Mulher } \\
\text { Gestora; Identidade; } \\
\text { Gênero; Poder. }\end{array}$ \\
\hline 9 & $\begin{array}{l}\text { Tese } \\
\text { *o trabalho }_{\text {não possui }} \\
\text { divulgação } \\
\text { autorizada }\end{array}$ & $\begin{array}{l}\text { HERRERA, Vânia } \\
\text { Érica }\end{array}$ & 2019 & $\begin{array}{l}\text { A vitrine da inclusão e o } \\
\text { espetáculo de Nicolau: a } \\
\text { ascensão profissional da } \\
\text { mulher acadêmica em cargos } \\
\text { de gestão em instituições de } \\
\text { ensino superior no Brasil. }\end{array}$ & $\begin{array}{l}\text { Mulheres; Barreiras } \\
\text { de gênero; Vitrine da } \\
\text { Inclusão; Espetáculo } \\
\text { de Nicolau; Gestão } \\
\text { do Ensino Superior. }\end{array}$ \\
\hline
\end{tabular}

Fonte: Site CAPES, organizado pelas autoras, 2021.

$\mathrm{Na}$ base de dados da CAPES, usando o descritor "mulheres gestoras", obtivemos 20 resultados, contemplando inúmeros campos do conhecimento. Explanando os resumos dos resultados encontrados, selecionamos nove títulos que enfatizaram o descritor "mulheres gestoras" na pesquisa pela relevância. Separamos as dissertações de Moraes (2014), Mafra (2015), Viana (2015) e Azevedo (2015), devido às singularidades no objeto de estudos e considerando o nível de estresse como gestoras no polo industrial e os mecanismos utilizados para enfrentamentos das causas.

Os conflitos de gênero e as estratégias a serem tomadas, para romper com questões de desigualdade construídas culturalmente pela sociedade a respeito da atuação feminina em gestão de empresas privadas, podem ser verificados nos estudos de Carneiro (2018) e Biasoli (2018). Podemos atrelar essa construção cultural ao que Chartier (2002) caracteriza por representações, 
sendo um instrumento de construção da realidade, inserida num ambiente social que compreende o mundo por meio das suas práticas culturais.

O reconhecimento da mulher como gestora vai além de questões relacionadas ao gênero, pois muitos são os empecilhos que restringem sua participação em cargos de alto escalão. Por mais qualificação profissional que possam ter, existe certo demérito quanto à capacidade de gerir, podendo ser evidenciado nos estudos de Teixeira (2018), Pimenta (2019) e Herrera (2019). As autoras refletem a dificuldade do progresso profissional feminino em instituições de Ensino Superior, por meio da discriminação, do preconceito e das relações de poder a serem enfrentadas.

Dos estudos apresentados, sete estão vinculados aos campos da Administração, indicando em seus resumos questões como: níveis de estresse no trabalho de mulheres gestoras e seus principais mecanismos de regulação; impactos na carreira de mulheres gestoras e estratégias de práticas para romper com as barreiras do tipo discriminação, preconceito e de gênero. Duas pesquisas mostram a ascensão de mulheres em cargos de gestão em instituições federais e como é construída essa identidade considerando a equidade de gênero e as dificuldades encontradas durante o percurso.

As pesquisas, como um todo, denotam que a participação da mulher em cargos de gestão nem sempre é bem-vista pelo seu campo de desempenho e pela sociedade, em que ainda são perceptíveis indícios de machismo e subalternidade, advindos da relação de poder. Às vezes, como retratam alguns trabalhos, se torna um processo árduo com alto nível de estresse. Alguns estudos a respeito da mulher na gestão em instituições educacionais indicam que, por mais qualificadas que sejam, algumas desistem do cargo em decorrência das barreiras encontradas durante o percurso profissional.

O resultado da busca com os outros três descritores foram semelhantes ao encontrado na base anterior, ou seja, nenhuma produção encontrada. Por mais que o primeiro descritor utilizado durante a busca na base de dados (mulheres gestoras) apontasse pesquisas que salientaram a presença de mulheres em cargos de gestão em instituições, na procura pelo descritor "mulheres gestoras na instituição" acabou-se não localizando nenhuma pesquisa vinculada à temática.

Resumindo a pesquisa por teses e dissertações nos bancos de dados da BDTD e da CAPES, resultou-se nos periódicos expostos na Tabela 1. 
Tabela 1 - Classificação de periódicos encontrados na bdtd e na capes

\begin{tabular}{l|c|c|c|c}
\hline \multicolumn{1}{c|}{ Descritores: } & $\begin{array}{c}\text { Banco de dados } \\
\text { BDTD }\end{array}$ & $\begin{array}{c}\text { Banco de dados } \\
\text { CAPES }\end{array}$ & Duplicados & Total \\
\hline $\begin{array}{l}\text { Mulheres } \\
\text { gestoras }\end{array}$ & 5 & 9 & 3 & 11 \\
\hline $\begin{array}{l}\text { Mulheres } \\
\text { gestoras na } \\
\text { educação }\end{array}$ & 0 & 0 & 0 & 0 \\
\hline $\begin{array}{l}\text { Mulheres } \\
\text { gestoras na } \\
\begin{array}{l}\text { História da } \\
\text { educação }\end{array}\end{array}$ & 0 & 0 & 0 & 0 \\
\hline $\begin{array}{l}\text { Mulheres } \\
\text { gestoras na } \\
\text { instituição }\end{array}$ & 0 & 0 & 0 & 0 \\
\hline Fonte: As autoras, 2021. & & & & \\
\hline
\end{tabular}

Comparando as duas bases de dados, a pesquisa tipo "estado da arte" resultou em 14 produções acadêmicas. Desse montante, três são comuns nas bases utilizadas. As 11 restantes se caracterizaram no descritor "mulheres gestoras", anulando as demais. O mapeamento possibilitou uma visão geral das pesquisas em relação à presença de mulheres gestoras, considerando os espaços em geral, da educação, da história da educação e nas instituições.

Foi nítido observar que grande parte dos trabalhos encontrados se enquadram no campo de conhecimento da administração, precisamente seis investigações que mostram os desafios encontrados com o cargo. Mesmo assim, as que restaram fazem menção direta à presença de mulheres gestoras em instituições de Ensino Superior. Apenas uma dissertação relata a trajetória de vida de duas professoras universitárias que atuaram como gestoras, intitulada como Professoras-gestoras na universidade: Reflexões a Partir das Trajetórias de Vida de Maria Carmem e Gaia (TEIXEIRA, 2018), vinculada ao Programa de Pós-graduação em Administração.

\section{Considerações finais}

A pesquisa tipo "estado da arte" possibilitou uma visão geral dos estudos a respeito da presença de mulheres gestoras em um período de cinco anos, considerando as áreas em geral, da educação, da história da educação e instituições. Foi nítido observar que grande parte dos trabalhos encontrados se enquadram no campo de conhecimento da administração, mesmo assim os que restaram fazem alguma menção direta ou indireta à presença de mulheres gestoras em instituições de ensino. Pode-se dizer que a maior dificuldade relatada pelas gestoras é o estresse causado tanto na caminhada para exercer a função, como também para se manter nela.

O que se observou é que os três últimos descritores, ao anularem a busca por não ter sido encontrado nenhum resultado, deixaram uma indagação em relação à educação. Por que não 
haveria trabalhos sobre gestão em educação escolar? Sabendo que as mulheres são a maioria em instituições de ensino, qual seria o motivo para não serem gestoras, ou, ao menos, o porquê de não serem objetos para estudos acadêmicos? Esses questionamentos surgem para refletirmos que ainda existem estereótipos criados culturalmente, inferiorizando a capacidade feminina de liderança de alto escalão. A desigualdade de gênero em pleno século XXI ainda aflige as mulheres que lutam constantemente pelo empoderamento de igualdade de direitos ao mesmo tempo em que nos instigam a prosseguir no caminho de investigação a respeito desse assunto no campo da gestão escolar.

Em vista disso, a contribuição desse "estado da arte" acerca dos descritores da mulher gestora na instituição, na escola, no ensino superior vem contribuir sobremaneira ao incentivo de pesquisa nessa área, em virtude de muitas mulheres atuarem em instituições da educação, gerindo projetos, tomando e implementando decisões e políticas públicas, entre outras.

Dessa forma, há a necessidade de estudos em relação à mulher em instituições de ensino básico e superior, que vem ao encontro do fortalecimento da participação e da promoção de acesso das mulheres contidas na Declaração Mundial Sobre Educação Superior no Século XXI em seu artigo 4. ${ }^{\circ}$, inciso c, o qual declara que: "Devem ser fomentados os estudos de gênero (ou estudos sobre a mulher) como campo específico de conhecimento, que tem um papel estratégico na transformação da educação superior e da sociedade." (UNESCO, 2009, grifo do original).

Em virtude da relevância do tema e de poucas pesquisas comparativas realizadas, conforme dados de periódicos da CAPES e da BDTD, a originalidade e a contribuição deste ensaio fazemnos refletir a respeito da importância das pesquisas em relação às mulheres, principalmente as mulheres que exercem cargos de gestoras em instituições educacionais, para que possam avançar nos estudos desde tema, dando ênfase a discussões e debates de relevâncias social, econômica, científica e acadêmica.

\section{Referências}

AZEVEDO, Erika. Estresse no trabalho: estudo com gestoras de organizações privadas do sul do estado de Minas Gerais. 2015. Vi, 160 f. Dissertação (Mestrado) - Centro Universitário Unihorizontes, 2015. Disponível em:

https://sucupira.capes.gov.br/sucupira/public/consultas/coleta/trabalhoConclusao/viewTrabal hoConclusao.jsf?popup=true\&id_trabalho=2980802. Acesso em: 10 set. 2020.

BIASOLI, Patricia Klaser. Mulheres em cargos de gestão: conciliações, dificuldades e conquistas. 2018. Vi, 167 f. Tese (Doutorado) - Universidade Federal do Rio Grande do Sul, 2018. Disponível em: https://sucupira.capes.gov.br/sucupira/public/consultas/coleta/trabalhoConclusao/view'Trabal hoConclusao.jsf?popup $=$ true\&id_trabalho $=6891778$. Acesso em: 10 set. 2020. 
CARNEIRO, Luziberto Barrozo. Teto de Vidro: um estudo sobre os fatores deste fenômeno no Brasil sob a percepção das mulheres gestoras. 2018. Vi, 108 f. Dissertação (Mestrado) Universidade Federal do Rio Grande, 2018. Disponível em:

https://sucupira.capes.gov.br/sucupira/public/consultas/coleta/trabalhoConclusao/viewTrabal hoConclusao.jsf?popup=true\&id_trabalho=6391222. Acesso em: 10 set. 2020.

CHARTIER, Roger. A história Cultural entre práticas e representações. Rio de Janeiro: Bertrand Brasil, 2002.

FERREIRA, Norma Sandra de Almeida. As pesquisas denominadas "estado da arte". Educação \& Sociedade, Campinas, v. 23, n. 79, p. 257-272, 2002. Disponível em:

scielo.br/scielo.php?pid=s0101-73302002000300013\&script=sci_abstract\&tlng=pt. Acesso em: 24 ago. 2020.

HERRERA, Vânia Érica. A vitrine da inclusão e o espetáculo de Nicolau: a ascensão profissional da mulher acadêmica em cargos de gestão em instituições de ensino superior no Brasil. 2019. Vi, 186 f. Tese (Doutorado) - Pontifícia Universidade Católica de São Paulo, 2019. Disponível em: https://tede2.pucsp.br/bitstream/handle/22669/2/V\%c3\%a2nia \%20\%c3\%89rica\%20Herrera.p df. Acesso em: 10 set. 2020.

MAFRA, Adriana Pereira Santos. Estresse no trabalho: estudo com gestoras do comércio varejista na região metropolitana de Belo Horizonte. 2015. Vi, 146 f. Dissertação (Mestrado) - Centro Universitário Unihorizontes, 2015. Disponível em:

https://sucupira.capes.gov.br/sucupira/public/consultas/coleta/trabalhoConclusao/viewTrabal hoConclusao.jsf?popup=true\&id_trabalho=3017215. Acesso em: 10 set. 2020

MORAES, Ana Flavia Moraes. Estresse ocupacional: um olhar sobre o trabalho da mulher gestora do Polo Industrial de Manaus. 2014. Vi, 159 f. Tese (Doutorado) - Universidade Federal de Minas Gerais, 2014. Disponível em: https:// repositorio.ufmg.br/handle/1843/BUBD-9VJFGV. Acesso em: 10 set. 2020.

PERROT, Michelle. Os excluídos da história: operários, mulheres e prisioneiros. Rio de Janeiro: Paz e Terra, 2017.

PIMENTA, Pauline Freire. "Lugar de mulher é na reitoria”: análise discursivo-crítica das formações identitárias e das relações de poder de mulheres do alto escalão nas IFES mineiras. 2019. Vi, 242 f. Tese (Doutorado) - Universidade Federal de Minas Gerais, 2019. Disponível em: epositorio.ufmg.br/bitstream/1843/30792/1/tese_final_PaulinePimenta.pdf. Acesso em: 10 set. 2020.

ROMANOWSKI, Joana Paulin. As licenciaturas no Brasil: um balanço das teses e dissertações dos anos 90. Tese (Doutorado) - Faculdade de Educação da Universidade de São Paulo, São Paulo, 2002

ROMANOWSKI, Joana Paulin; ENS, Romilda Teodora. As Pesquisas Denominadas do Tipo "Estado da Arte" em educação. Diálogo Educacional, v. 6, n. 19, p. 37-50, 2006. Disponível em: file://C:/Users/Nootbook/Desktop/TCC/Textos-Referêncial Teórico/diálogo-237.pdf.

Acesso em: 21 ago. 2020. 
SILVA, Eliane Vanildo da. Gênero e ensino superior: a inserção das mulheres nos cursos de engenharias da UNESC. 2019. 177 f. Dissertação (Mestrado) - Universidade do Extremo Sul Catarinense, 2019. Disponível em: http://repositorio.unesc.net/handle/1/7633. Acesso em: 10 set. 2020.

SILVA, Kátia Elaine de Vasconcelos e. A representatividade das mulheres na gestão acadêmica da Universidade Federal de Pernambuco. 2018. Dissertação (Mestrado) - Universidade Federal de Pernambuco, 2018. Disponível em: https:/ / repositorio.ufpe.br/handle/123456789/30649. Acesso em: 10 set. 2020.

SPINK, P. Análise de documentos de domínio público. In: SPINK, M. J. (Org.). Práticas discursivas e produção de sentido. São Paulo: Cortez, 1999. p. 123-152.

UNESCO. Conferência Mundial sobre Ensino Superior 2009. As novas dinâmicas do Ensino Superior e pesquisas para a mudança e o desenvolvimento social. Comunicado. Paris: UNESCO, 2009

VIANA, Elisete Pereira Goncalves. Estresse Ocupacional: estudo com mulheres gerentes que atuam em uma instituição bancária na cidade de Belo Horizonte-MG. 2015. Dissertação (Mestrado) - Centro Universitário Unihorizontes, 2015. Disponível em:

https://sucupira.capes.gov.br/sucupira/public/consultas/coleta/trabalhoConclusao/viewTrabal hoConclusao.jsf?popup=true\&id_trabalho=3088729. Acesso em 10 set. 2020.

TEIXEIRA, Marcella Barbosa Miranda. Professoras-Gestoras na Universidade: reflexões a partir das trajetórias de vida de Maria Carmen e Gaia. 2018. Dissertação (Mestrado) - Centro Federal de Educação Tecnológica de Minas Gerais, 2018. Disponível em:

https://sucupira.capes.gov.br/sucupira/public/consultas/coleta/trabalhoConclusao/viewTrabal hoConclusao.jsf?popup=true\&id_trabalho=7176812. Acesso em: 10 set. 2020. 